\title{
Effet de l'incorporation de la noix de cajou dans les rations alimentaires sur les performances de croissance des porcs : phases post-sevrage et de croissance
}

\author{
Koffi Sylvanus Aubert YAO*, Moussa KIMSE, Dofara SORO et Agathe FANTODJI \\ Université Nangui-Abrogoua, 02 BP 801 Abidjan 02, Abidjan, Côte d'Ivoire. \\ *Auteur correspondant; E-mail : k.sylvio79@yahoo.fr, Tel. : (+225) 45466096
}

\section{RESUME}

Quatre types d'aliments dont le taux d'incorporation de noix est variable ont été formulés pour les stades post-sevrage et de croissance. Ces aliments ont été testés sur 120 porcs (Large White x Piétrain) x (Landrace x Duroc) par stade physiologique. En post-sevrage, il n'y a eu aucun effet de l'anacarde sur les paramètres de croissance de l'animal. En croissance, le poids vif des animaux ayant reçu les rations à 7 et $9 \%$ d'anacarde a baissé de 2 à $3 \mathrm{~kg}$ environ par rapport à ceux de la ration à $0 \%$ d'anacarde. Le gain moyen quotidien (GMQ) du lot à $9 \%$ d'anacarde est environ $30 \mathrm{~g} / \mathrm{j}$ inférieur au lot sans anacarde. Les aliments contenant 7 et $9 \%$ d'anacarde sont environ $65 \mathrm{~g} / \mathrm{j}$ moins ingérés que celui ne contenant pas d'anacarde $(\mathrm{P}<0,05)$. L'anacarde n'a pas eu d'effet sur l'indice de consommation aux stades physiologiques étudiés de l'animal. En ce qui concerne la santé de l'animal, l'anacarde n'a eu aucun impact négatif sur les animaux. Néanmoins, en phase de croissance, le taux de créatinine pour les lots à $9 \%$ d'anacarde baisse de $3 \mathrm{mg}$ en moyenne par rapport au lot à $0 \%$ d'anacarde $(\mathrm{P}<0,05)$.

(C) 2013 International Formulae Group. All rights reserved.

Mots clés : Porc, noix de cajou, croissance, santé, stade physiologique.

\section{INTRODUCTION}

Les graines agroindustrielles constituent une source alimentaire majeure chez le porc (Ironkwe et Amefule, 2008). Grâce à leur richesse en protéine et en énergie, elles jouent un rôle essentiel dans la croissance des animaux. Elles favorisent la réduction du déficit en protéine et en énergie tout en permettant une réduction du coût alimentaire (Royer et al., 2005). C'est le cas $\mathrm{du}$ tourteau de soja, des graines entières de pois, de soja dont l'utilisation dans les rations alimentaires améliore significativement la croissance et l'indice de consommation chez les porcelets (Albar et al., 2000 ; Burnham et al., 2000, Bertol et al., 2001). Ainsi, tout comme les graines précédemment citées, l'incorporation de la noix de cajou (Anacardium occidentale) dans l'alimentation du bétail a présenté des avantages. Introduite sous forme farineuse dans les rations alimentaires des poulettes de 21 à 28 semaines d'âge, la noix de cajou n'a pas affecté l'âge pour les premières pontes et le poids des œufs malgré une ingestion alimentaire réduite de $8,9 \%$ par rapport à la ration sans noix (Odunsi, 2002). A. occidentale est une graine issue de la famille des anacardiaceae qui s'adapte à des régimes pluviométriques très 
divers. Elle est sensible au froid et à l'altitude (Son et Traoré, 2002 ; MINAGRA (Ministère de l'Agriculture et des Ressources Animales), 2004). L'analyse chimique révèle que la teneur moyenne d'énergie métabolisable (Energie de la fraction des aliments effectivement digérés diminuée de celle représentée par les urines) et de protéine des résidus de ces noix de cajou obtenus à la suite du concassage et du décorticage de l'amande, sont respectivement de $4882,66 \mathrm{kcal}$ et de $21 \%$ (Lacroix, 2003). La noix de cajou peut être donc une source de protéine et d'énergie appréciable pour l'alimentation animale. En Côte d'Ivoire, ces résidus de noix de cajou, de par leur accessibilité régulière et leur coût relativement bas (60 F CFA le kg estimé après une étude de marché non publiée), ont été proposés pour l'alimentation porcine en vue de combler le déficit protéique et énergétique de l'aliment. Cependant, cette idée, selon les informations en notre possession, a été envisagée sans que des études préalables soient menées sur leur effet chez le porc. Le présent travail a pour but d'évaluer l'effet du niveau d'incorporation de la noix de cajou sur la croissance du porc durant les phases de démarrage (post-sevrage) et de croissance de celui-ci en Côte d'Ivoire.

\section{MATERIEL ET METHODES}

L'objectif de l'étude est de déterminer l'influence de la noix de cajou (anacarde) sur les performances de croissance des porcs et quelques indicateurs physiologiques sanitaires chez le porc. Quatre lots d'animaux ont été sélectionnés et nourris avec des aliments formulés à base d'anacarde.

\section{Animaux et aliments}

120 porcs mâles castrés et femelles confondus (64 mâles castrés et 56 femelles) issus de croisement (Large White $x$ Piétrain) $\mathrm{x}$ (Landrace $\mathrm{x}$ Duroc) ont été sélectionnés par stade physiologique. Ces animaux ont été élevés dans des loges individuelles de dimensions $1,5 \mathrm{~m}^{2} \times 2,10 \mathrm{~m}$, de température $26,4 \pm 2,5^{\circ} \mathrm{C}$ et $75 \%$ d'humidité relative.
Quatre lots de 30 animaux (16 mâles castrés et 14 femelles dans le même lot) ont été constitués aux stades physiologiques étudiés de l'animal. Chacun des 4 lots a été nourris $a d$ libitum à l'aide d'un aliment spécifique différent les uns des autres par le taux d'incorporation de la noix de cajou ( $A$. occidentale) séché. La composition chimique des aliments a été déterminée par analyse bromatologiques (Tableau 1). Deux distributions ont été effectuées au cours d'une journée, une $1^{\text {ère }}$ à $8 \mathrm{~h}$ du matin après le $1^{\mathrm{er}}$ nettoyage des loges, une $2^{\text {nde }}$ à $14 \mathrm{~h}$ faisant suite au $2^{\text {nd }}$ nettoyage de la journée. En postsevrage, entre 6 et 12 semaines d'âge, les 4 lots animaux ont reçu respectivement l'aliment à $0 \%$ de noix de cajou pour le lot $\mathrm{D}_{0}$, l'aliment contenant $2 \%$ de noix de cajou pour le lot $\mathrm{D}_{2}$, l'aliment à $4 \%$ de noix de cajou pour le lot $\mathrm{D}_{4}$ et l'aliment à $6 \%$ de noix de cajou pour le lot $\mathrm{D}_{6}$. Pendant la croissance entre 12 et 24 semaines d'âge, chacun des 4 lots a été alimenté à l'aide de l'aliment $\mathrm{C}_{0}, \mathrm{C}_{5}, \mathrm{C}_{7}$ et $\mathrm{C}_{9}$ contenant respectivement $0,5,7$ et $9 \%$ de noix de cajou.

\section{Récolte de l'anacarde}

Les résidus d'anacarde utilisés sont des rejets de noix de cajou issus du traitement de celles-ci en vue de l'extraction de l'amande qu'elle contient. Ils ont été récoltés dans la zone de Dimbokro (Côte d'Ivoire) à $200 \mathrm{~km}$ environ d'Abidjan par la société OLAM Ivoire SARL (Société de production et de commercialisation de produits agricoles: cajou, coton et café-cacao en Côte d'Ivoire). Les noix récoltées ont été séchées à la température ambiante. Les impuretés sur la graine ont été ensuite éliminées par nettoyage à sec, les graines ont été réhumidifiées par un trempage dans de l'eau pendant 1 heure au moins selon les recommandations MCF. (1980). Les graines ont été amenées dans un bain de baume à $185{ }^{\circ} \mathrm{C}$ pendant 90 secondes et ensuite essorées pour l'élimination du baume utilisé. Après l'essorage, les graines ont été concassées et décortiquées en vue d'extraire l'amande de cajou de la coque. Les 
résidus de graines de noix de cajou utilisés dans ce travail sont issus de cette étape de concassage et de décorticage mais ayant échappés au décorticage. Elles représentent environ 20 à $30 \%$ des graines traités.

\section{Elevage et mesures zootechniques}

Le poids moyen des animaux a été relevé en début de chaque stade physiologique en vue de l'obtention des poids initiaux à l'aide d'une balance de type $\mathrm{EKS}^{\circledR}$, de capacité $100 \mathrm{~kg}$ et de précision $100 \mathrm{~g}$. L'effet de la ration sur la croissance a été mesuré par des relevés hebdomadaires des poids individuels des animaux sur toute la durée de l'élevage. Ces pesées ont été réalisées dans la matinée avant la distribution de l'aliment. Le suivi de l'ingéré a été effectué par des pesées quotidiennes de la quantité d'aliment distribuée et du refus à l'aide d'une balance de marque $\mathrm{EKS}^{\circledR}$, de capacité $5 \mathrm{~kg}$ et de précision $1 \mathrm{~g}$.

\section{Impact de l'aliment sur la santé}

Les animaux ont fait l'objet d'inspection quotidienne en vue de déceler les éventuels morbides caractérisés par un aspect nonchalant et un refus de s'alimenter. Des prélèvements de sang ont été effectués pour une étude physiopathologique. Ainsi, $5 \mathrm{ml}$ de sang ont été prélevés dans un tube sous vide bouchonné VENOSAFE $^{\circledR}$ de $6 \mathrm{ml}$, dimension: 16 x $100 \mathrm{~mm}$, à l'aide d'une aiguille DECTON DICKSON VACUTAINERE SYSTEM $^{\circledR}$, de précision glide, référence 360748 , dimension : 1,2 x 38 $\mathrm{mm}$, au niveau de la veine jugulaire de tous les animaux à jeun à chaque stade physiologique et en fin d'élevage. Les prélèvements ont été conservés à $4^{\circ} \mathrm{C}$ dans un récipient thermostaté COLEMAN POLYLITE $40^{\circledR}$ moins de $12 \mathrm{~h}$ avant les différents dosages au laboratoire. Les échantillons ont été centrifugés à $3000 \mathrm{G}$ et le sérum récupéré dans des tubes de $5 \mathrm{ml}$ pour le dosage de l'urée, la glycémie, la créatinine, le cholestérol total, le triglycéride, le phosphore et le calcium. Ces dosages ont été réalisés à l'aide d'un spectromètre de marque BIOLABO-BIOCARE $^{\circledR}$.

\section{Analyses Statistiques}

Les données zootechniques et sanitaires ont été analysées par le test ANOVA (analyses de variances) à partir du logiciel STASTISCA 6. La comparaison des moyennes, afin d'évaluer l'impact de l'apport de la noix de cajou sur les performances des animaux, a été effectuée à l'aide du test de Tukey.

\section{RESULTATS}

Aucun cas de trouble digestif (diarrhées et constipations éventuelles recherchées) ni de mortalité n'a été observé au cours de l'essai. Toutefois, 11 cas d'infections bactériennes (pneumonies et broncho-pneumonies, en général) ont été détectés et traités à l'aide d'antibiotiques (Oxytétracycline 20\% et PenTrep).

\section{Impact de l'anacarde sur les performances zootechniques}

Aucune différence significative n'a été observée entre la croissance des males castrés et celle des femelles de façon générale. En post-sevrage, entre le sevrage et 12 semaines d'âge, l'incorporation d'anacarde n'a eu aucun effet significatif sur la croissance et sur l'efficacité alimentaire (Tableau 2). Le poids, le GMQ, l'ingéré et l'indice de consommation moyens observés durant cette période sont respectivement de $15,5 \mathrm{~kg}, 183 \mathrm{~g} / \mathrm{j}, 485 \mathrm{~g} / \mathrm{j}$ et 2,65 .

En croissance, entre 12 et 24 semaines d'âge par contre, l'incorporation d'anacarde entraine une baisse de la croissance. Le poids vif à 24 semaines d'âge est d'environ 2 et $3 \mathrm{~kg}$ plus bas chez les animaux ayant reçu les rations contenant respectivement 7 et $9 \%$ d'anacarde par rapport à ceux dont la ration n'en possédait pas $(\mathrm{P}<0,05)$. 
K. S. A. YAO et al. / Int. J. Biol. Chem. Sci. 7(2): 479-488, 2013

Tableau 1: Composition des régimes (g/100 g).

\begin{tabular}{|c|c|c|c|c|c|c|c|c|}
\hline \multirow[t]{3}{*}{ Ingrédients } & \multicolumn{8}{|c|}{ Stades physiologiques } \\
\hline & \multicolumn{4}{|c|}{ Post-sevrage } & \multicolumn{4}{|c|}{ Croissance } \\
\hline & $\mathbf{D}_{0}$ & $\mathbf{D}_{2}$ & $\mathbf{D}_{4}$ & $\mathbf{D}_{6}$ & $\mathrm{C}_{0}$ & $\mathrm{C}_{5}$ & $\mathbf{C}_{7}$ & $\mathrm{C}_{9}$ \\
\hline Anacarde & 0 & 2 & 4 & 6 & 0 & 5 & 7 & 9 \\
\hline Maïs & 55,1 & 53,1 & 51,1 & 49,1 & 52,0 & 47,0 & 45,0 & 43,0 \\
\hline Son de blé & 12 & 12 & 12 & 12 & 11 & 11 & 11 & 11 \\
\hline Farine basse & - & - & - & - & 10 & 10 & 10 & 10 \\
\hline Tourteau de coprah & 15 & 15 & 15 & 15 & 15 & 15 & 15 & 15 \\
\hline Tourteau de soja & 8 & 8 & 8 & 8 & 5 & 5 & 5 & 5 \\
\hline Farine de poisson & 7 & 7 & 7 & 7 & 4 & 4 & 4 & 4 \\
\hline Coquillage & 1 & 1 & 1 & 1 & 1,5 & 1,5 & 1,5 & 1,5 \\
\hline Sel & 0,5 & 0,5 & 0,5 & 0,5 & 0,5 & 0,5 & 0,5 & 0,5 \\
\hline CMV & 1,4 & 1,4 & 1,4 & 1,4 & 1 & 1 & 1 & 1 \\
\hline Totaux & 100 & 100 & 100 & 100 & 100 & 100 & 100 & 100 \\
\hline \multicolumn{9}{|l|}{ Composition chimique (\%) } \\
\hline Matière sèche & 89,5 & 92,4 & 90,3 & 89,7 & 91,9 & 92,7 & 89,6 & 90,1 \\
\hline Protéine & 17,8 & 18,07 & 18,14 & 18,21 & 15,91 & 16,18 & 16,25 & 16,39 \\
\hline Matière grasse & 4,15 & 5,25 & 5,90 & 6,65 & 4,44 & 6,10 & 7,21 & 8,00 \\
\hline Fibres brutes & 4,71 & 4,77 & 4,74 & 4,81 & 5,3 & 4,92 & 4,88 & 4,85 \\
\hline Calcium & 0,97 & 0,89 & 0,90 & 0,93 & 0,92 & 0,85 & 0,87 & 0,89 \\
\hline Phosphore total & 0,65 & 0,60 & 0,61 & 0,61 & 0,66 & 0,52 & 0,53 & 0,55 \\
\hline Acides gras saturés & 41,6 & 39,4 & 36,65 & 32,46 & 40,2 & 36 & 32,48 & 30,45 \\
\hline Acides gras insaturés & 58,4 & 61,6 & 63,35 & 65,54 & 59,8 & 64 & 67,52 & 69,55 \\
\hline Energie métabolisable (kcal) & 2912,5 & 2920,3 & 2926,5 & 2937,5 & 2800 & 2830,2 & 2860,5 & 2890,1 \\
\hline
\end{tabular}


Tableau 2: Effet du taux d'incorporation de la noix de cajou dans la ration sur l'ingestion, les performances de croissance et l'efficacité alimentaire chez le porc durant les phases post-sevrage et de croissance.

\begin{tabular}{|c|c|c|c|c|c|c|c|c|}
\hline \multirow[t]{3}{*}{ Paramètres de croissance } & \multicolumn{8}{|c|}{ Stades physiologiques } \\
\hline & \multicolumn{4}{|c|}{ Post-sevrage } & \multicolumn{4}{|c|}{ Croissance } \\
\hline & $\mathbf{D}_{0}$ & $\mathbf{D}_{2}$ & $\mathbf{D}_{4}$ & $\mathbf{D}_{6}$ & $\mathbf{C}_{0}$ & $\mathbf{C}_{5}$ & $\mathbf{C}_{7}$ & $\mathrm{C}_{9}$ \\
\hline Ingestion alimentaire $(\mathrm{g} / \mathrm{j})$ & $488,3 \pm 55,3$ & $487,7 \pm 61,2$ & $483,7 \pm 55,3$ & $480,1 \pm 59,6$ & $1690 \pm 91^{\mathrm{a}}$ & $1654 \pm 80^{\mathrm{ab}}$ & $1632 \pm 80^{b}$ & $1615 \pm 72^{b}$ \\
\hline Indice de consommation & $2,6 \pm 0,4$ & $2,64 \pm 0,5$ & $2,67 \pm 0,4$ & $2,70 \pm 0,5$ & $2,9 \pm 0,2$ & $2,91 \pm 0,2$ & $2,91 \pm 0,2$ & $2,93 \pm 0,2$ \\
\hline Gain Moyen Quotidien (g/j) & $188,3 \pm 18,6$ & $184,3 \pm 20,5$ & $180,7 \pm 14,4$ & $177,4 \pm 20,4$ & $583,9 \pm 42,7^{\mathrm{a}}$ & $568,3 \pm 25,3^{\mathrm{ab}}$ & $560,8 \pm 46,7^{\mathrm{ab}}$ & $550,1 \pm 32,8^{\mathrm{b}}$ \\
\hline Poids Vif Moyen (kg) & $15,8 \pm 1,2$ & $15,7 \pm 1,1$ & $15,5 \pm 1,1$ & $15,4 \pm 1,4$ & $64,3 \pm 3,1^{\mathrm{a}}$ & $62,9 \pm 2,6^{\mathrm{ab}}$ & $62,4 \pm 2,9^{b}$ & $61,5 \pm 2,4^{b}$ \\
\hline
\end{tabular}

abcd les moyennes du même stade physiologique affectées de lettre différentes sont significativement différents $(P<0,05)$. 
Tableau 3 : Effet du taux d'incorporation de la noix de cajou dans la ration sur quelques indicateurs physiopathologiques chez le porc durant les phases post-sevrage et de croissance.

\begin{tabular}{|c|c|c|c|c|c|c|c|c|}
\hline \multicolumn{9}{|c|}{ Stades physiologiques } \\
\hline \multirow[t]{2}{*}{ Paramètres sanguins } & \multicolumn{4}{|c|}{ Post-sevrage } & \multicolumn{4}{|c|}{ Croissance } \\
\hline & $\mathbf{D}_{0}$ & $\mathbf{D}_{2}$ & $\mathbf{D}_{4}$ & $\mathbf{D}_{6}$ & $\mathrm{C}_{0}$ & $\mathrm{C}_{5}$ & $\mathrm{C}_{7}$ & $\mathrm{C}_{9}$ \\
\hline Urée (g/l) & $0,27 \pm 0,05$ & $0,30 \pm 0,04$ & $0,25 \pm 0,06$ & $0,31 \pm 0,05$ & $0,27 \pm 0,05$ & $0,32 \pm 0,05$ & $0,27 \pm 0,05$ & $0,30 \pm 0,04$ \\
\hline Glycémie (g/l) & $0,65 \pm 0,08$ & $0,71 \pm 0,09$ & $0,68 \pm 0,06$ & $0,66 \pm 0,08$ & $0,70 \pm 0,06$ & $0,68 \pm 0,05$ & $0,65 \pm 0,06$ & $0,64 \pm 0,07$ \\
\hline Créatinine (mg/l) & $15,28 \pm 1,55$ & $15,30 \pm 1,90$ & $15,38 \pm 1,16$ & $15,19 \pm 1,84$ & $19,32 \pm 2,19^{\mathrm{a}}$ & $17,49 \pm 2,85^{\mathrm{ab}}$ & $17,17 \pm 2,19^{\mathrm{ab}}$ & $16,36 \pm 2,73^{b}$ \\
\hline Cholestérol total (g/l) & $1,02 \pm 0,19$ & $1,09 \pm 0,21$ & $1,05 \pm 0,12$ & $1,07 \pm 0,16$ & $1,08 \pm 0,15$ & $1,03 \pm 0,19$ & $0,97 \pm 0,16$ & $1,06 \pm 0,12$ \\
\hline Triglycéride (g/l) & $0,24 \pm 0,06$ & $0,23 \pm 0,03$ & $0,25 \pm 0,05$ & $0,24 \pm 0,04$ & $0,24 \pm 0,05$ & $0,25 \pm 0,05$ & $0,27 \pm 0,07$ & $0,24 \pm 0,05$ \\
\hline Calcium (mg/l) & $81,21 \pm 8,20$ & $82,19 \pm 7,29$ & $84,57 \pm 8,31$ & $82,71 \pm 8,81$ & $85,20 \pm 9,25$ & $83,35 \pm 8,40$ & $84,26 \pm 7,50$ & $83,82 \pm 8,71$ \\
\hline Phosphore (mg/l) & $54,70 \pm 7,36$ & $54,60 \pm 8,44$ & $55,23 \pm 8,12$ & $53,82 \pm 7,45$ & $55,45 \pm 8,24$ & $54,52 \pm 8,33$ & $54,51 \pm 7,05$ & $56,40 \pm 8,35$ \\
\hline
\end{tabular}

abcd les moyennes du même stade physiologique affectées de lettre différentes sont significativement différents $(P<0,05)$ 
Les gains moyens quotidiens sont toutefois égaux à l'exception du lot à $9 \%$ d'anacarde dans la ration dont la moyenne de gain quotidien est environ $30 \mathrm{~g} / \mathrm{j}$ inférieur au lot sans anacarde $(\mathrm{P}<0,05)$. Le GMQ moyen des 3 lots à 0,5 et $7 \%$ d'anacarde est de 570 $\mathrm{g} / \mathrm{j}$. Les aliments contenant 7 et $9 \%$ d'anacarde sont environ $65 \mathrm{~g} / \mathrm{j}$ moins ingérés à celui ne contenant pas d'anacarde $(\mathrm{P}<0,05)$. Le taux de $5 \%$ d'anacarde n'entraine pas de modification par rapport à $0 \%(1672 \mathrm{~g} / \mathrm{j}$ en moyenne). L'incorporation de l'anacarde dans la ration en croissance n'entraine pas non plus de variation de l'indice de consommation. La moyenne générale observée est de 2,91 (Tableau 2).

\section{Impact de l'anacarde sur les paramètres physiopathologiques}

En post-sevrage, entre 6 et 12 semaines d'âge, l'incorporation d'anacarde n'a eu aucun effet significatif sur les paramètres physiopathologiques de l'animal. Les taux moyens observés d'urée, de glycémie, de créatinine, de cholestérol, de triglycéride, de calcium et de phosphore durant cette période sont respectivement de 0,28 g/l, 0,68 g/l, 15,3 $\mathrm{mg} / \mathrm{l}, 1,05 \mathrm{~g} / \mathrm{l}, 0,24 \mathrm{~g} / \mathrm{l}, 82 \mathrm{mg} / \mathrm{l}$ et de $54 \mathrm{mg} / \mathrm{l}$ (Tableau 3).

En phase de croissance, l'incorporation de l'anacarde n'a eu aucune influence sur l'urée, la glycémie, le cholestérol, le triglycéride, le calcium, le phosphore et également sur le taux de créatinine à 5 et $7 \%$ d'anacarde dans la ration. Par contre, pour la ration à $9 \%$ d'anacarde, le taux de créatinine baisse de $3 \mathrm{mg}$ en moyenne par rapport à la ration sans anacarde $(\mathrm{P}<0,05)$. Les taux moyens d'urée, de glycémie, de cholestérol, de triglycéride, de calcium, de phosphore et de créatinine des lots à 0,5 et $7 \%$ d'anacarde sont respectivement de 0,29 g/l, 0,67 g/l, 1,04 $\mathrm{g} / \mathrm{l}, 0,25 \mathrm{~g} / \mathrm{l}, 84 \mathrm{mg} / \mathrm{l}, 55 \mathrm{mg} / \mathrm{l}$ et de $18 \mathrm{mg} / \mathrm{l}$.

\section{DISCUSSION}

En post-sevrage, l'absence d'effet significatif de l'incorporation de l'anacarde dans la ration sur l'ingestion alimentaire observé dans le présent travail confirme les résultats obtenus sur un autre oléoprotéagineux notamment le soja (Fekete et al., 1987). Ces auteurs ont observé qu'avec des rations contenant 10 et $20 \%$ de graines de soja extrudées, il n'y a aucune variation de l'ingestion alimentaire chez les porcelets sevrés. La teneur stable en énergie maintenue dans ces régimes, consécutive au taux relativement bas de matières grasses (40 à 60 $\mathrm{g} / \mathrm{kg}$ de matière sèche) dans ces rations expliquerait cette invariabilité de l'ingestion alimentaire selon ces auteurs. Ce qui est par ailleurs le cas dans cet essai avec des proportions de matière grasse de 5,2 à $6,7 \%$ dans les rations contenant l'anacarde pendant la phase post-sevrage. Cette absence de différence significative de l'ingestion pourrait expliquer en partie l'équivalence de croissance et de l'efficacité alimentaire observée dans ce travail. Ces résultats sont, également, concordant avec ceux de Reis de Souza et al. (1990) qui notent que pour un taux d'incorporation de $20 \%$ de graine de colza dans les rations, le gain de poids et l'indice de consommation ne sont pas affectés chez les porcelets $2^{\text {ème }}$ âge. Cependant ces résultats sont en partie en contradiction avec ceux de Fekete et al. (1987) qui bien que n'ayant pas observé d'effet significatif de l'apport de soja sur l'ingestion des animaux, ont constaté une amélioration de la croissance et l'indice de consommation. Ceux-ci ont obtenu qu'avec des rations à 10 et $20 \%$ de graines de soja extrudées ayant une proportion basse de matières grasses (40 à $60 \mathrm{~g} / \mathrm{kg}$ de matière sèche), une hausse respective de 14 et $29 \mathrm{~g}$ du gain de poids et une réduction de l'indice de consommation de 0,09 unité pour la ration à $20 \%$ par rapport au témoin. 
En croissance, la diminution des paramètres de croissance, notamment l'ingestion, le gain moyen quotidien et le poids vif observée dans cette étude ne confirme pas les résultats obtenus dans d'autres travaux antérieurs avec certaines graines oléo-protéagineuses. En effet, les travaux, effectués en France, de Albar et al. (1992) pour une incorporation de $25 \%$ de graine de pois, et ceux de Albar et al. (2000) pour un taux d'incorporation de 4 et $8 \%$ de graine de tournesol oléique dans les rations alimentaires pour porcs en engraissement (entre 23 et $110 \mathrm{~kg}$ de poids vifs) ont montré que, l'ingestion alimentaire et le gain moyen quotidien ne sont pas modifiés. Toutefois, en accord avec nos résultats, l'indice de consommation n'a pas varié. Pour ces auteurs, cette invariabilité des paramètres de croissance est, également, due aux faibles proportions de matières grasses (4 à 5\%) dans les rations. Ce qui n'est pas le cas du taux relativement élevé de matières grasses $(7,21$ à $8 \%$ ), en phase de croissance, obtenu au cours de cette étude après incorporation de 7 et $9 \%$ d'anacarde. Des taux qui pourraient expliquer la baisse de l'ingestion alimentaire. En effet, une teneur croissante en énergie due à une proportion élevée de matières grasses et particulièrement d'acides gras insaturés dans une ration provoque une baisse de l'ingestion de l'aliment en raison de leur inappétence par le porc (Atteh et al., 1992; Leeson et al., 1997). Ce qui serait liée à leur digestibilité moyenne (Noblet et al., 2008) et pourrait être un risque laxatif pour l'intestin grêle (Mourot et Lebret, 2009). Par contre, pour d'autres auteurs tels que Fetuga et al. (1974) et Mba et al. (1974), la diminution de la consommation pourrait être liée à un meilleur équilibre des acides aminés contenus dans la protéine de la noix de cajou. Cette baisse de l'ingestion entraine également la chute de la croissance des animaux.
En croissance, la baisse du taux de créatinine liée à des proportions élevées de noix de cajou (9\%) dans l'aliment confirme les conclusions de Tietz (1999) qui stipulent que la quantité de créatinine produite chaque jour est fonction de la masse musculaire. La diminution $\mathrm{du}$ poids vif des animaux consécutif à l'augmentation des proportions d'anacarde dans les rations entrainerait donc une baisse de la production de créatine.

La plupart des paramètres sanguins n'ayant pas variés dans les différents stades physiologiques des animaux confirment les résultats de Flipot et al. (1983). En effet, ces auteurs n'ont pas noté de changement significatif de la concentration de quelques paramètres sanguins (calcium et phosphore) dans les états physiologiques de l'animal après une incorporation de $10 \%$ de tourteau de canola dans les rations alimentaires du porc. Par contre, pour ce même taux d'incorporation de tourteau de canola, les concentrations de la plupart des paramètres sanguins (le phosphore, l'urée, le sodium, le potassium) ont significativement augmenté avec les états physiologiques. Ce qui n'est pas le cas dans cette étude où les paramètres sanguins n'ont pas varié avec les états physiologiques où des valeurs identiques ont été observées en postsevrage et en croissance sauf pour les taux de créatinine. Une augmentation du taux de creatinine qui serait liée à l'augmentation de la masse musculaire des animaux du post sevrage à la croissance (Tietz, 1999). En effet, un profil métabolique stable (transformations très peu variable dans l'organisme), maintenu par chaque animal et en rapport à la quantité d'aliment ingérée, expliquerait cette invariabilité des paramètres sanguins avec les états physiologiques (Flipot et al., 1983).

Aussi, les paramètres physiopathologiques évalués pour les lots sans anacarde et ceux avec l'anacarde aux différents stades physiologiques correspondent aux normes exigées pour une 
bonne santé de l'animal (Vaissaire et al., 1976). L'utilisation de l'anacarde dans les rations alimentaires ne présente donc aucun effet néfaste sur la santé de l'animal.

\section{Conclusion}

L'étude de l'incorporation de la noix de cajou (anacarde) dans les rations alimentaires sur les paramètres de croissance du porc révèle qu'au stade post-sevrage, l'anacarde n'a eu aucun effet sur la croissance et la santé de l'animal. En phase de croissance, l'introduction de l'anacarde à 7 et $9 \%$ dans les rations a fait baisser l'ingéré alimentaire et le poids vif chez l'animal. Le gain moyen quotidien n'a été influencé qu'à $9 \%$ d'anacarde dans la ration. Par contre, l'indice de consommation n'a pas subi de variation. Pour les paramètres de santé, seul le taux de créatinine a connu une baisse à $9 \%$ d'anacarde.

L'incorporation de l'anacarde dans les rations alimentaires conduirait alors, à obtenir des porcs de moins en moins lourds, par une baisse de l'ingéré et du gain moyen quotidien sans toutefois baisser l'indice de consommation et sans être néfaste pour leur santé. L'usage de la noix de cajou, de façon progressive à une certaine proportion, en substitution du maïs pourrait être bénéfique pour l'éleveur en lui permettant de faire des économies par la réduction de ses coûts alimentaires.

Par ailleurs, ces expérimentations ont été menées qu'à deux stades physiologiques de l'animal. Il conviendrait donc d'envisager d'autres études au stade de finition de l'animal afin d'avoir une idée plus nette de l'influence de l'incorporation de ces noix de cajou sur la croissance des porcs.

Des études sur la digestibilité et la fermentescibilité des fibres après incorporation de la noix de cajou pourraient, également être envisagées afin d'évaluer le niveau d'acceptation des aliments.

\section{REFERENCES}

Albar J, Chauvel J, Granier R. 1992. About the use of spring field peas in dry or wet feeding for fattening pigs. Journées Recherches Porcine, 24: 179-186.

Albar J, Royer E, Massabie P, Mourot J, Vendeuvre JL. 2000. The use of oleic acid-rich sunflower seed in diets for growing pigs: effects on backfat quality. Journées Recherches Porcine, 32: 297303.

Atteh JO, Balogun OO, Annogu AA, Kolade MA. 1992. Replacement value of maize milling waste for maize in the diet of growing pullets. Tropical Agriculture, 70: 267-270.

Bertol I, Beutler JF, Leite D, Batistela O. 2001. Propriedades físicas de um Cambissolo Húmico afetadas pelo tipo de manejo do solo. Science Agricola, 58: 555-560.

Burnham DA, Derstler KL, Currie PJ, Bakker RT, Zhou Z, Ostrom JH. 2000. Remarkable new birdlike dinosaur (Theropoda: Maniraptora) from the Upper Cretaceous of Montana. University of Kansas Paleontological Contributions, 13 : 1-14.

Fekete J, Gatel F, Castaing J, Seroux M. 1987. Valeur alimentaire pour des régimes à base d'orge enrichis avec des matières grasses pour porcelets sevrés. Journées Recherches Porcine, 19: 303-310.

Fetuga BL, Babatunde GM, Oyenuga VA. 1974a. Composition and nutritive value of cashew nut to rats. Journal of Agriculture and Food Chemistry, 22: 678-680.

Flipot P, Tremblay AV, Dufour JJ. 1983. Effet, sur quelques paramètres sanguins, de 1'addition de tourteau de canola CV, tower à la ration des truies. Canadian Veterinary Medical Association, 24: 278280. 
Ironkwe MO, Amefule KU. 2008. Appraisal of indigenous pig procution and management practices in rivers state. Nigeria Journal of Agriculture and Social Research (JASR), 8(1): 1-7.

Lacroix E. 2003. Les Anacardiers, les Noix de Cajou et la Filière Anacarde à Bassila et au Bénin, Projet Restauration des Ressources Forestières de Bassila. GFA Terra Systems Eulenkrugstraße 82 22359: Hamburg, Allemagne; 75p.

Leeson S, Caston L, Summers JD. 1997. Layer performance of 4 strains of Leghorns subjected to various rearing programmes. Poultry Science, 76: 1-5.

Mba AV, Njike MC, Oyenuga VA. 1974. The proximate composition and the amino acid content of some Nigerian oilseeds. Journal Sciences Food Agriculture, 25: 1547-1553.

MCF (Ministère de la Coopération Française). 1980. Mémento de l'Agronome. Collection Techniques Rurales en Afrique; 1600p.

MINAGRA (Ministère de l'Agriculture et des Ressources Animales). 2004. Filière Anacarde, Situation Actuelle et Perspective de Développement. Edition TPC; 19p.

Mourot J, Lebret B. 2009. Modulation de la quantité de la viande de porc par l'alimentation. INRA Productions Animales, 22(1): 33-40.

Noblet J, Jaguelin-Peyraud Y, Quemeneur B, Chesneau G. 2008. Valeur energétique de la graine de lin chez le porc: impact de la technologie de caisson-extrusion. Journées Recherches Porcine, 40: 203208.

Odunsi AA, 2002. Effect of feeding rejects cashew kernel meal on pre and earlylaying performance of pullet. Archives Zootechnic, 5: 423-429.

Reis de Souza T, Melcion JP, Bourdon D, Giboulot G, Peiniau JA, Aumaitre A, 1990. La graine de colza crue ou extrudée : une nouvelle source d'énergie et de protéine dans l'alimentation des porcelets. Journées Recherches Porcine, 22: 151-158.

Royer E, Chauvel J, Courboulay V, Granier R, Albar J. 2005. Oléo-protéagineux : quel taux d'incorporation en post-sevrage et engraissement? Technique Porc, 28(1): 13-19.

Son G, Traoré S. 2002. Analyse du secteur de l'anacarde: situation actuelle et perspective de développement. Rapport effectué au nom du Centre du commerce international CNUCED/OMC (CCI) Burkina Faso. Fonds global d'affectation spéciale du CCI dans le cadre du projet INT/W3/69: 12p.

Tietz NW. 1999. Text Book of Clinical Chemistry $\left(3^{\text {rd }}\right.$ Edn) Burtis CA, Ashwood ER, Saunders WB; 1241-1245.

Vaissaire J, Renault L, Palisse M, Linder Th, Maire CL, Labadie JP. 1976. Biochimie sanguine et électrophorèse des protéines sériques intérêt en pathologie porcine. Journées Recherches Porcine, 8: 251258. 